Sous prétexte de contrôler les factures, les assureursmaladie veulent avoir accès au plus grand nombre possible de données des patients, naturellement aussi à celles qui sont confidentielles. La révision du codage et le modèle genevois montrent très nettement qu'il est possible d'effectuer des contrôles gradués efficaces sans violer le secret médical, primordial pour le traitement des patients. Deux exemples à suivre!

Dr Gert Printzen, membre du Comité central

\title{
Cybersanté et protection des données: qui est finalement responsable des patients?
}

\begin{abstract}
Dans la cybersanté, la protection des données est toujours plus sujette à caution. Les assureurs-maladie aboliraient complètement le secret médical s'ils le pouvaient. A l'ère du «tout informatique», ils exigent un accès aux données du dossier patient électronique équivalent à celui des médecins. Et, dans le système DRG, ils demandent l'indication systématique et détaillée des diagnostics et des procédures ainsi que d'autres informations médicales (minimal data set) sur la facture. Leur but avoué est de «déceler à temps l'évolution potentiellement négative d'une maladie et de convenir de mesures efficaces avec l'assuré concerné» [1]. En d'autres termes, les assureurs-maladie aimeraient réduire les coûts en s'immisçant dans le traitement. Ils se voient comme des gestionnaires de soins, de préférence comme dans la LAA où l'assureur-accidents peut ordonner au patient le traitement à suivre. Est-ce le rôle que le monde politique aimerait confier aux assureurs-maladie? Non, car le Parlement a par exemple explicitement refusé la transmission des données demandées sous le système DRG [2].

Le conseiller fédéral Couchepin a critiqué la transmission exigée par les assureurs dans le cadre du minimal data set en l'estimant disproportionnée: «Nous sommes convaincus que la solution du Conseil fédéral et de la majorité est suffisante pour permettre aux assureurs de faire leur travail,
\end{abstract}

\section{Il est possible d'effectuer des contrôles sans violer la protection des données.}

(...) et nous pensons aussi que, dans le doute, il faut trancher en faveur de la protection des données plutôt qu'en faveur du confort des assureurs-maladie, confort relatif puisqu'ils recevraient tellement d'informations qu'ils ne seraient plus en état de les maîtriser.» [3]. Le conseiller national, et rapporteur de la commission, Yves Guisan est allé encore plus loin: «De plus, la gestion de cette masse impressionnante de données va (...) engendrer aussi des coûts supplémentaires absolument considérables. Cela correspond à une conception manifestement dépassée des contrôles. (...) Si l'on accepte la proposition de la minorité (...), c'est la fin du système du médecin-conseil.» [4].

Lors du vote qui a suivi, les députés ont rejeté la transmission systématique des procédures et diagnostics pertinents. Que s'est-il passé depuis?

En Allemagne, les coûts administratifs pour le contrôle des factures DRG s'élèvent à 350 euros par cas. En Suisse, tel ou tel assureur a débauché des codeurs hospitaliers et met sur pied ses propres services. Il faudrait à l'avenir que les assureurs développent fortement leurs services de médecinconseil et leur infrastructure informatique.

Les assureurs disposent depuis plus d'une décennie des données du «minimal data set» sous forme anonymisée. Néanmoins, à ce jour, ils ne se sont pas encore donné la peine de montrer comment ils entendent utiliser ces données, et encore moins de dire pourquoi ils en ont besoin. Les critères EAE semblent donc ne pas valoir pour les assureurs.

La révision du codage par des tiers externes est le seul instrument permettant de contrôler efficacement le codage. Il fait maintenant partie du paysage suisse.

Les Hôpitaux universitaires de Genève et les assureurs ont institué une procédure correspondant aux critères de la protection des données et à la législation actuelle. Ainsi, à Genève, les diagnostics et les procédures codés ainsi que les rapports d'hospitalisation les plus importants ne sont transmis qu'à la demande expresse de l'assurance et à chaque fois exclusivement au médecin-conseil. Seules quelque 20 à 30 factures sur plus des 43000 établies chaque année sont corrigées suite à une correction du codage. D'ailleurs, le chiffre des cas pour lesquels les assureurs ont demandé le dossier médical a baissé de $20 \%$ en deux ans.

Malgré tout, les caisses-maladie ne se lassent pas de continuer à demander les données médicales. Et aujourd'hui, l'OFSP semble vouloir mettre en œuvre, par le biais de conventions tarifaires, ce que le Parlement a explicitement rejeté.

Et pourtant, la révision du codage et l'exemple genevois montrent qu'il est parfaitement possible d'effectuer des contrôles gradués efficaces dans le respect du secret médical - primordial pour le traitement des patients.

Judith Wagner, Dr sc. hum., responsable eHealth de la FMH Hanspeter Kuhn, avocat, secrétaire général adjoint de la FMH

1 Zellweger U. Helsana AG. 8.2. Leistungsabwicklung in der Krankenversicherung - automatisierte Verarbeitung von Leistungen. In: W. Oggier et al. Gesundheitswesen Schweiz im Umbruch

2 Kuhn HP. Arrêt du 29 mai 2009: pas de décision phare pour l'introduction de SwissDRG. Bull Méd Suisses. 2011;92(16/17):605.

3 BO $2007 \mathrm{~N} 444 \mathrm{~s}$

4 BO 2007 N 445. 